\section{FDA approves first drug for Parkinson disease psychosis}

Although dopamine replacement strategies can alleviate the motor symptoms of Parkinson disease, patients have had few therapeutic options for non-motor symptoms, which include depression, dementia, sleep abnormalities and autonomic failure. Now the FDA has approved Acadia Pharmaceuticals' pimavanserin as the first drug to treat hallucinations and delusions associated with psychosis in Parkinson disease, which affect up to $50 \%$ of patients during the course of their illness.

Pimavanserin, a serotonin (5-hydroxytryptamine) receptor $2 \mathrm{~A}\left(5-\mathrm{HT}_{2 \mathrm{~A}}\right)$ inverse agonist, failed in its first three randomized and controlled trials, showing no improvement on the primary end point of the Scale for the Assessment of Positive Symptoms (SAPS), a scale that was designed for schizophrenia. The developers refined the end point before launching a fourth trial, focusing the scale on the items of most clinical relevance for Parkinson disease. This trial showed that the drug decreases the frequency and/or severity of hallucinations and delusions without worsening the primary motor symptoms of Parkinson disease. In March, the FDA's panel of independent psychopharmacological drug experts voted 12 to 2 in favour of approval, although many of the experts said that the drug's efficacy was only modest. The most common side effects include swelling, nausea and confusion.

Analysts forecast that annual global sales for pimavanserin could hit US $\$ 2.8$ billion by 2021 , shows data from Thomson Reuters Cortellis.

Other experimental drugs aimed at non-motor aspects of Parkinson disease include Biotie Therapies' SYN-120, a dual 5- $\mathrm{HT}_{6}$ and 5- $\mathrm{HT}_{2 \mathrm{~A}}$ antagonist, which is in Phase lla trials for cognitive dysfunction associated with Parkinson disease.

Two adenosine $A_{2 A}$ receptor inhibitors, Biotie's tozadenant and Kyowa Hakko Kirin Co.'s istradefylline, are in Phase III trials for their potential to treat both motor and non-motor aspects of the degenerative disease. However, Merck \& Co. previously made it to Phase III trials with its adenosine $A_{2 A}$ receptor antagonist preladenant, but failed to generate evidence of efficacy.

Asher Mullard

\section{Tackling antimicrobial drug resistance}

Antibiotic drug development is fraught with scientific risk and business challenges (Nat. Rev. Drug Discov. 14, 529-542; 2015 and Nat. Rev. Drug Discov. 13, 711-713; 2014). Two recent reports have laid out plans to tackle both these hurdles.

A scientific roadmap by the Pew Charitable Trusts lists four sets of scientific priorities. These include the need for new chemical matter that might lead to more success with antibacterial discovery. "One key challenge is that commercially available chemical matter is not well suited for antibiotic discovery given that the physicochemical properties of antibiotics are unique," they write, before suggesting a multi-step plan to selectively generate and modify chemical matter for the discovery of new antibiotics. They also argue that proof-of-concept studies are needed to enable the development of "nontraditional' therapies, like antivirulence strategies, monoclonal antibodies against bacterial targets and combination approaches.
A second 18-month review of the antimicrobial resistance (AMR) landscape, commissioned by the prime minister of the United Kingdom and chaired by economist Jim O'Neill, took a broader perspective. The final report from this review suggests nine interventions, ranging from improved sanitation to increased R\&D funding. On the funding front, they write that "there is insufficient private and public investment in R\&D in support of new drugs and other areas relevant to the global AMR challenge. The funding that does exist is not always as focused and coordinated (particularly across national borders) as it could be to maximize its impact." They therefore called for the creation of a Global Innovation fund for AMR, endowed with US $\$ 2$ billion over 5 years. They also call for 'market entry rewards' - of around $\$ 800$ million to $\$ 1.3$ billion - for sponsors who successfully develop new antibiotics for prospectively defined unmet needs.

O'Neill and his colleagues estimate that the total cost of global action on AMR is about $\$ 40$ billion over a decade. They note that "the investment needed to take action is dwarfed by the human and financial cost of inaction which is mounting already." They propose a few possible ways to pay for the necessary initiatives, including the reallocation of a fraction of global funding from international institutions to AMR, and the application of an antibiotic investment charge to pharmaceutical companies who do not invest in AMR research.

Asher Mullard

\section{NCATS launches 'periodic table' for medicinal product ingredients}

As drug development and production has become an increasingly globalized process, regulators and industry stakeholders have struggled to efficiently and accurately exchange information about what substances are in any given medicine. The NIH's National Center for Advancing Translational Sciences (NCATS) is now launching the Global Ingredient Archival System (GINAS) to facilitate this process. "Instead of relying on drug or chemical names, which vary across countries and regions, GINAS will enable substances to be defined by standardized, scientific descriptions," says the NCATS. GINAS classifies substances according to broad categories (chemical, protein, plant substance, etc.) and captures details for each substance category consistent with international standards (chemical structure, DNA sequence, etc).

NCATS scientists developed the software for this system with input from the FDA, the European Medicines Agency and other regulatory authorities. Regulators will use this software to store the detailed, proprietary information they need to fulfil their regulatory function. NCATS has published a public subset of these data on its website. NCATS will release a new public version - with information on medicinal products, uses and metabolism of substances, mechanisms of action and more - towards the end of the year.

Asher Mullard

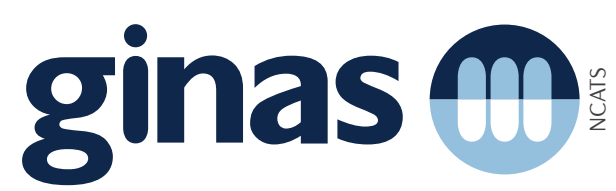

